\title{
Personalising Vibrotactile Displays through Perceptual Sensitivity Adjustment
}

\author{
Granit Luzhnica \\ Know-Center \\ Graz, Austria \\ gluzhnica@know-center.at
}

\author{
Viktoria Pammer \\ Graz Univ. of Technology \\ Graz, Austria \\ viktoria.pammer@tugraz.at
}

\author{
Sebastian Stein \\ University of Glasgow \\ Glasgow, UK \\ sebastian.stein@glasgow.ac.uk
}

\author{
Eduardo Veas \\ Know-Center \\ Graz, Austria \\ eveas@know-center.at
}

\author{
John Williamson \\ University of Glasgow \\ Glasgow, UK \\ jhw@dcs.gla.ac.uk
}

\author{
Roderick Murray Smith \\ University of Glasgow \\ Glasgow, UK \\ rod@gla.ac.uk
}

\begin{abstract}
Haptic displays are commonly limited to transmitting a discrete set of tactile motives. In this paper, we explore the transmission of real-valued information through vibrotactile displays. We simulate spatial continuity with three perceptual models commonly used to create phantom sensations: the linear, logarithmic and power model. We show that these generic models lead to limited decoding precision, and propose a method for model personalization adjusting to idiosyncratic and spatial variations in perceptual sensitivity. We evaluate this approach using two haptic display layouts: circular, worn around the wrist and the upper arm, and straight, worn along the forearm. Results of a user study measuring continuous value decoding precision show that users were able to decode continuous values with relatively high accuracy (4.4\% mean error), circular layouts performed particularly well, and personalisation through sensitivity adjustment increased decoding precision.
\end{abstract}

\section{ACM Classification Keywords}

H.5.2 Information Interfaces and Presentation: User Interfaces-Haptic I/O

\section{Author Keywords \\ haptic feedback; tactile feedback; haptic display; phantom sensation; wearable; encoding information; user study; HCI}

\section{INTRODUCTION}

Haptic displays attract increasing interest in the research community and are investigated in a wide range of application areas. These include augmented [25] and virtual reality [32], human-computer interaction $[5,19,38,50]$, navigation aids

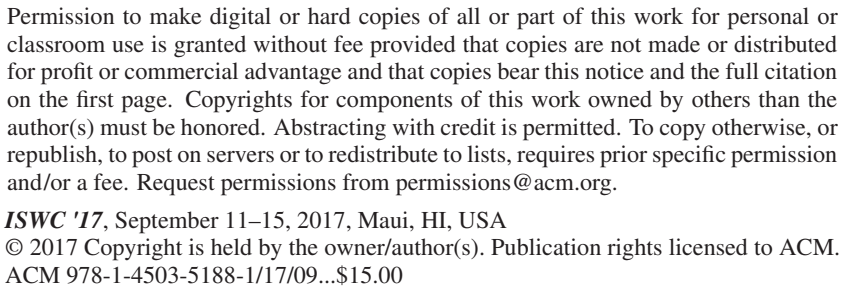
classroom use is granted without fee provided that copies are not made or distributed for profit or commercial advantage and that copies bear this notice and the full citation on the first page. Copyrights for components of this work owned by others than the author(s) must be honored. Abstracting with credit is permitted. To copy otherwise, or republish, to post on servers or to redistribute to lists, requires prior specific permission and/or a fee. Request permissions from permissions@acm.org.

ISWC '17, September 11-15, 2017, Maui, HI, USA

(C) 2017 Copyright is held by the owner/author(s). Publication rights licensed to ACM. ACM 978-1-4503-5188-1/17/09...\$15.00

https://doi.org/10.1145/3123021.3123029
$[10,11,12,36,41,43,45]$, and assistive technology for people with impaired vision [4, 16, 30, 49], hearing [15], speech [24], or vestibular function [2, 23, 47], and for people with stroke, Parkinson's disease, spinal cord injury or peripheral neuropathy $[13,17,40]$. A large body of prior work on vibrotactile displays has investigated the transmission of a discrete set of tactile motives such as caller ids [5], alphanumerical characters [4, 16, 27, 30, 49], and simple shapes [48]. Real-valued haptic feedback would be useful for transmitting continuous quantities such as grasp force, task progress, joint rotation angle, or movement speed.

Displays targeting other senses have long established guidelines for representing quantitative real-valued data grounded in psychophysics. For instance, visual features that people preattentively quantify and compare have been found to be suitable for providing visual feedback on task progress [28]. Such clear guidelines are presently missing for encoding real-valued quantities for haptic feedback. Tactile stimuli can encode information by varying stimulation amplitude, frequency, duration or location [27, 42, 44, 49]. Amplitude-modulated and frequency-modulated tactile stimuli have previously shown to yield relatively low decoding accuracy [8, 31, 42]. In this paper, we investigate peoples' decoding precision of spatially modulated real-valued data.

The challenge of creating spatially continuous vibrotactile feedback is to imbue a continuous sensation with a limited, small number of tactors (vibration motors). In previous work, the phantom effect has been used to create the illusion of spatial continuity [7, 21, 38, 39]. It is an interpolation effect that occurs when two tactors are active, and the user attributes perception to a location in-between the active tactors due to an inherently low resolution of haptic perception [1]. Evaluation of spatially continuous tactile displays has so far mainly focused on the quality of perceived continuity, the consistency of perceived intensity across space, and the perceived smoothness of time-varying continuous movements.

In this paper, we investigate user decoding precision of spatially modulated real-valued data, which is decoded by localising spatially continuous, temporally stationary (static) 
vibrotactile stimuli. In a comprehensive user study, we quantify decoding precision across two vibrotactile display layouts (circular, wornaround the wrist and the upper arm, and straight, worn along the forearm) each positioned at two different locations on the body. In each testing condition, we evaluate three perceptual models of phantom sensation from the literature with respect to localisation precision: the linear model, the log model, and the power model. Our main contribution is a data-driven method for haptic display personalisation. We propose to adjust perceptual models to idiosyncratic and spatial variations in perceptual sensitivity learned from user-specific data, and quantitatively compare localisation precision using all perceptual models with and without sensitivity adjustment.

\section{RELATED WORK}

Starting with Braille's invention of the Braille coding in 1824, haptic displays have long been widely used by people with visual impairments, and research on tactile displays equipped with actuators has been ongoing since at least 1924 [15].

Alphanumeric information has been encoded through a combination of variations in amplitude, frequency, duration and locations of stimuli $[18,16,42,44,49]$. Recently, Nicolau [30] mapped Braille to tactors on six fingers of both hands. Liao [26] constructed a display composed of an array of $2 \times 2$ tactors on the wrist to encode letters and numbers using a spatiotemporal encoding. Luzhnica [27] used a prioritised overlapping spatiotemporal encoding of six tactors to encode the English alphabet. In shape printing, symbols are encoded with a low spatial resolution using an array of tactile stimulators. White [48] transmit images captured by a camera through a $20 \times 20$ array of tactile vibrators, enabling participants to recognise simple object shapes (circle, square and triangle) after training. Bliss [4] used a video camera and a $6 \times 24$ matrix of vibrators placed on the users' fingers to convert letters to tactile stimuli. Gault [15] used a piezoelectric unit named "telectactor" to redirect captured speech. Kirman [24] transmitted speech streams to the palm using a $15 \times 15$ vibrator matrix. Six participants could successfully discriminate 15 different words. Wall [2] transmit head orientation and tilt through a vibrotactile array on the shoulders and the trunk for people with vestibular loss. Wall and Kentala [47, 23] transmit posture information from motion sensors to vibrotactile arrays on the torso. A similar setup as been proposed for correcting body posture [17] and arm posture [13] after stroke. For people with hand prostheses, Chatterjee [8] and Pylatiuk [34] used frequency modulation to encode levels of grasp force, as did Sanders [37] using an array of eight tactors on the forearm.

Phantom sensation has been studied in the literature primarily with a focus on users' perceived quality of temporally dynamic continuous stimuli $[38,39,7,14,20]$. These studies indicate that the log model provides a qualitatively good movement sensation, as the vibration intensity is perceived as more stable towards the middle between two tractors compared to the linear model. Results of [39] suggest, however, that the linear model may yield higher localisation precision between two tactors compared to the log model. Similarly, the results of [3] show that linear model could be a good choice for localisation of perceived stimuli. Recently, Israr [21] introduced the power model, which also maintains intensity across space and participants had no clear preference between the power model and the log model [38]. In this paper, we quantify participants' localisation precision comparing all three of these models and propose a data-driven method for model personalization to improve localisation precision.

Vibrotactile feedback with phantom sensation has been proposed for immersive experiences in multimedia [38], games [19], storytelling [51], augmented [25] and virtual reality $[32,25]$. Directional information has been transmitted through tactile displays in [46], where directions are encoded through continuous movement vectors and in [50], where a vibrotactile spherical device composed of 13 vibrotactile modules is proposed for navigation. As this paper proposes a method for improving spatial localisation precision in general, our contribution may help improve the immersiveness and quality of these tactile experiences in the future.

There is a large body of work in HCI using phantom sensation at different body locations including the forearms [7, 35], palm $[39,50]$, finger tips $[46,25]$ and the back [38]. While this paper focuses on the wrist, the forearm, and the upper arm, the proposed sensitivity adjustment is equally applicable to other locations.

\section{METHOD}

Human haptic perception has, depending on body location, a relatively low spatial resolution. Simultaneous stimulation of two or more locations in close proximity may only be perceived as a single stimulation somewhere in between tactors. This haptic illusion is typically referred to as phantom sensation [1]. The exact location of the perceived stimulus depends, among other factors, on the stimulation amplitudes [1, 38, 33]. This Section starts by briefly describing three perceptual models of phantom sensation from the literature. We then introduce our proposed method for personalisation, extending these generic models by explicitly modelling and accounting for idiosyncratic and spatial variation in perceptual sensitivity. We outline extending spatially continuous haptic displays to more than two tactons per dimension, and describe how we estimate local senstivity from user data.

\section{Perceptual Models of Phantom Sensation}

Perceptual models of phantom sensation for vibrotactile feedback can be described as a generative function $f: \mathscr{R}^{T} \rightarrow[0,1]$, mapping stimulation amplitudes of a fixed set of $T$ tactors onto a continuous location along one spatial axis at which stimulation is perceived. The inverse model $f^{-1}:[0,1] \rightarrow \mathscr{R}^{T}$ determines the set of stimulation amplitudes required to invoke a phantom sensation at a given location.

The amplitude with which tactors are stimulated in practice is bounded within an interval $\left[A_{\min }, A_{\max }\right]$ by minimum perception thresholds, hardware constraints, and user comfort. Consequently, we describe models with respect to normalized stimulation intensities $I \in[0,1]$, derived using Eq. (1)

$$
I=\frac{A-A_{\min }}{A_{\max }-A_{\min }}, \quad A=I\left(A_{\max }-A_{\min }\right)+A_{\min } .
$$




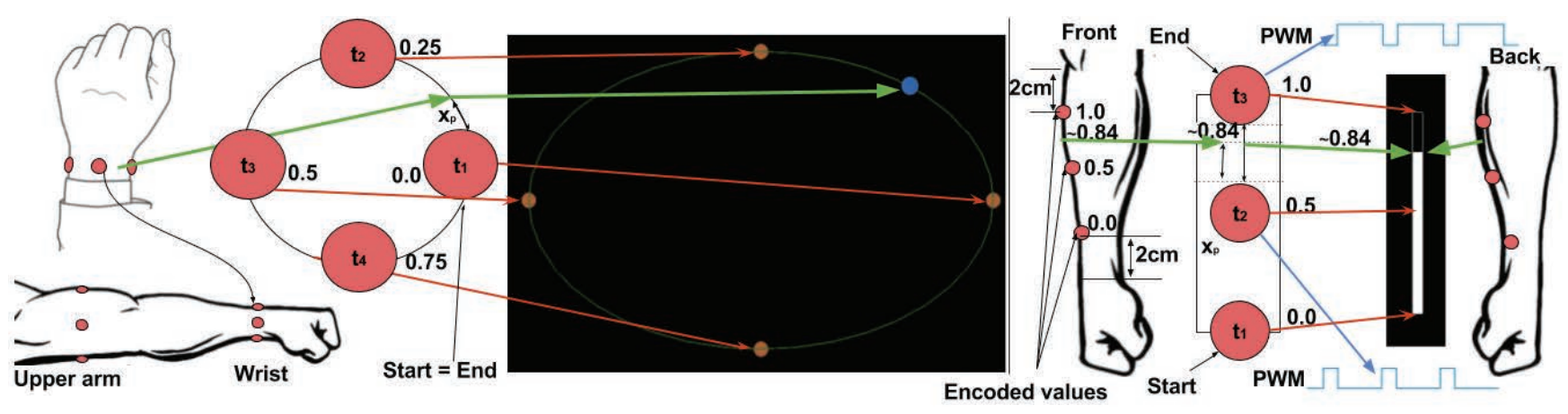

Figure 1: Circular layout, straight layout and their corresponding user interfaces used in the study (with black background). On the circular layout $t_{1}$ and $t_{2}$ are activated whereas on the straight one, $t_{2}$ and $t_{3}$ are activated (see also PWM signal) to encode the value.

Given a pair of tactors $i \in\{0,1\}$, actuated with intensities $I_{i}$ at locations $x_{i}=i$ along some spatial dimension, the location of the phantom sensation can be described as $x_{p} \in[0,1]$.

We consider the linear model, the log model and the power model $[1,21]$. In the linear model, shown in Eqns. (2), $x_{p}$ is proportional to the relative stimulation intensity of $I_{1}$ and, inversely, stimulation intensities are proportional to their proximity to $x_{p}$. In the log model, described by Eqns. (3) and (4), the stimulation intensities have a logarithmic relationship with perceived location $\left(x_{p}\right)$. The recently proposed power model $[21,38]$ is based on the energy summation of Pacinian channels [29]. Here, the perceived location is dependent on the square of stimulation intensities as in Eq. (5),

$$
\begin{gathered}
x_{p}^{l}=\frac{I_{1}}{I_{0}+I_{1}}, \quad I_{0}^{l}=\left(1-x_{p}\right), \quad I_{1}^{l}=x_{p} \\
I_{0}^{g}=\frac{\log \left(2-x_{p}\right)}{\log (2)}, \quad I_{1}^{g}=\frac{\log \left(1+x_{p}\right)}{\log (2)} \\
\left(1+x_{p}^{g}\right)^{I_{0}}=\left(2-x_{p}^{g}\right)^{I_{1}} \\
x_{p}^{p}=\frac{I_{1}^{2}}{I_{0}^{2}+I_{1}^{2}}, \quad I_{0}^{p}=\sqrt{1-x_{p}}, \quad I_{1}^{p}=\sqrt{x_{p}} .
\end{gathered}
$$

We construct $f\left(A_{0}, A_{1}\right)$ and $f^{-1}\left(x_{p}\right)$ by combining each model with Eq. (1). The phantom location $f\left(A_{0}, A_{1}\right)$ is estimated by mapping stimulation amplitudes to intensities using Eq. (1)(left) followed by estimating $x_{p}^{l}, x_{p}^{p}$ or $x_{p}^{g}$. Determining the stimulation amplitudes that create a phantom sensation $f^{-1}\left(x_{p}\right)$ involves estimating intensities $\left(I_{0}^{l}, I_{1}^{l}\right),\left(I_{0}^{p}, I_{1}^{p}\right)$ or $\left(I_{0}^{g}, I_{1}^{g}\right)$ and corresponding amplitudes using Eq. (1)(right).

\section{Sensitivity-adjusted Perceptual Models}

All models in the literature implicitly assume a constant stimulation sensitivity across locations on the body at which tactors are placed. They can, therefore, be considered as generic models. Cholewiak [9] provided evidence suggesting that there are fine-grained spatial differences in perceptual sensitivity on the forearm: the area around the wrist and upper part towards the elbow are more sensitive than the middle part of the forearm. As there are spatial variations in perceptual sensitivity, it is reasonable to expect that perceptual sensitivity also varies across users. One indication for this is provided by [35], showing strong gender-based differences in preference between the linear model and the log model. Therefore, we propose to create personalised perceptual models by explicitly incorporating user-specific measures of local sensitivity at tactor locations.

Intuitively, the higher the stimulation sensitivity at some location the lower the stimulation intensity needs to be to create a fixed intensity sensation. Thus, to account for spatial variations in perceptual differences of stimulation sensitivity, we propose to scale intensities $I_{i}$ with a user and location-specific scale factor $s_{i} \geq 1$. This local rescaling is independent of the perceptual model of phantom sensations and can, therefore, be applied to all three models as shown in Eqns. (6)-(8),

$$
\begin{gathered}
x_{p}^{l s}=\frac{s_{1} I_{1}}{s_{0} I_{0}+s_{1} I_{1}}, \quad I_{0}^{l s}=\frac{1-x_{p}}{s_{0}}, \quad I_{1}^{l s}=\frac{x_{p}}{s_{1}} \\
I_{0}^{g s}=\frac{\log \left(2-x_{p}\right)}{s_{0} \log (2)}, \quad I_{1}^{g}=\frac{\log \left(1+x_{p}\right)}{s_{1} \log (2)} \\
\left(1+x_{p}^{g s}\right)^{s_{0} I_{0}}=\left(2-x_{p}^{g s}\right)^{s_{1} I_{1}} \\
x_{p}^{p s}=\frac{s_{1}^{2} I_{1}^{2}}{s_{0}^{2} I_{0}^{2}+s_{1}^{2} I_{1}^{2}}, \quad I_{0}^{p s}=\frac{\sqrt{1-x_{p}}}{s_{0}}, \quad I_{1}^{p s}=\frac{\sqrt{x_{p}}}{s_{1}} .
\end{gathered}
$$

For the generic and personalised log models, Eqns. (4) and (8)), $f\left(A_{0}, A_{1}\right)$ can not be computed in closed form. Thus, optimization techniques should be used for finding $x_{p}^{g s}$.

After formalizing how models are extended to chains of $N>2$ tactors, we describe how sensitivity values $s_{i}$ can be estimated from data.

\section{Extension to Chains of $N>2$ Tactors}

When two tactors are further apart than a few inches (depending on their location on the body), the user senses two separate stimulations instead of one: one at each tactor location. With additional tactors, the area on the body can be increased without losing the phantom sensation. We explore vibrotactile displays with chains of three and four tactons.

Let us consider the general case of representing values $v \in$ $[0,1]$ with $N$ equidistant tactors $0 \leq i<N$ and $M$ segments between tactors. In order to apply Eqns. (2)-(9), we need to 
determine the relevant pair $(a, b)$ of tactors for stimulation and estimate the within-interval value $x_{p}$ from $v$. This is accomplished using Eq. (10), where \% is the modulo operator,

$$
a=\lfloor v M\rfloor \% N, \quad b=(a+1) \% N, \quad x_{p}=v M-a .
$$

Figure 1 illustrates a straight and a circular tactile display, the values $v$ corresponding to tactor locations, a phantom sensation and its distance to the two closest tactors.

\section{Data Driven Sensitivity Estimation}

While sensitivity values $s_{i}$ could naively be adjusted manually or pre-configured for different body positions, we propose to estimate them from calibration data. We consider a set of $D$ datapoints $\left\{\left(y_{j}, I_{1}^{j}, \ldots, I_{N}^{j}\right)\right\}$, where $y_{j} \in[0,1]$ is the stimulus location perceived by the user when tactors are set to stimulation intensities $I_{1}^{j}, \ldots, I_{N}^{j}$.

We express the problem of finding optimal sensitivities $S$ : $s_{1}, \ldots, s_{N}$ as a minimization problem of the mean squared error between encoded values and user responses:

$$
S_{o}=\underset{S}{\arg \min } \frac{1}{D} \sum_{j=1}^{D}\left(y_{j}-v\left(S, I_{1}^{j}, \ldots, I_{N}^{j}\right)\right)^{2}, \quad s_{i} \geq 1 .
$$

Note that this optimisation requires the evaluation of $v\left(S, I_{1}^{j}, \ldots, I_{N}^{j}\right) \in[0,1]$ in every step, which involves estimating $x_{p}$. While $x_{p}$ can be calculated in closed form for the linear model and the power model using Eqns. (6) and (9), finding $x_{p}$ for the log model involves solving one optimisation problem for every data point:

$$
x_{p o}=\underset{x_{p}}{\arg \min }\left(\left(1+x_{p}^{g s}\right)^{s_{a}^{j} I_{a}^{j}}-\left(2-x_{p}^{g s}\right)^{s_{b}^{j} I_{b}^{j}}\right)^{2}, 0<x_{p}<1 .
$$

We use the L-BFGS-B algorithm [6] for all optimisations, which is a quasi-Newton method that can handle simple bounding box constraints.

\section{USER STUDY}

A user study was conducted to quantify real-valued data decoding precision using spatial modulation of a stationary stimulus. Decoding precision was measured under twenty-four conditions: two display layouts, two body positions per display layout, and three perceptual models with and without personalisation through sensitivity adjustment.

\section{Participants}

Participants were recruited among students of a local technical University. Sixteen participants (all right handed, ten male, aged between 26 and 41, and six female, aged between 21 and 32) volunteered to take part in the study. Only one female participant had previously taken part in a study on haptics. Detailed participant characteristics are presented in Table 1.

\section{Apparatus}

An Arduino Due board (see Fig. 2) controlled a set of $3.4 \mathrm{~mm}$ vibrotactile motors of type ROB-08449 (Voltage range: $2.3 \mathrm{~V}-3.6 \mathrm{~V}$; Amplitude vibration: $0.8 G$ ). Instead of changing the vibration amplitude directly, different intensities of vibration are generated by varying PWM duty cycles.

\begin{tabular}{l||l|l|l|l} 
Gender & Age & Wrist & Upper Arm & Forearm \\
\hline \hline Male & $25.60(2.89)$ & $17.47(1.23)$ & $28.56(2.64)$ & $27.13(1.67)$ \\
\hline Female & $29.67(5.45)$ & $15.33(1.21)$ & $26.33(4.59)$ & $23.37(2.65)$ \\
\hline All & $27.12(4.48)$ & $16.67(1.60)$ & $27.72(3.64)$ & $25.72(2.77)$
\end{tabular}

Table 1: Participant characteristics (mean and standard deviation): Age, wrist circumference $(\mathrm{cm})$, upper arm circumference $(\mathrm{cm})$, and wrist to elbow forearm length $(\mathrm{cm})$.
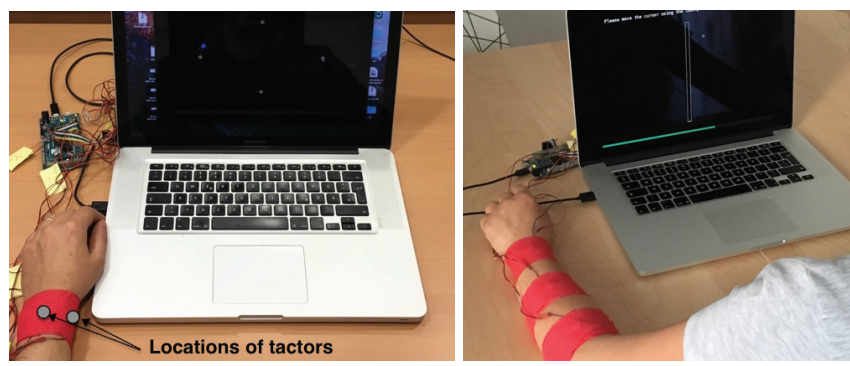

Figure 2: Participants performing the study on the wrist (left) and forearm (right). Note that the hand (left) is supinated relative to the rest position, which may result in user inputs being shifted.

We designed two vibrotactile display layouts for encoding realvalued data through spatial modulation. The circular layout (Fig. 2, left) with four equidistant tactors was worn around the wrist and the upper arm. We envisage this layout to be useful for encoding values that represent the state of repetitive or circular processes, orientation or angular information. The straight layout (Fig. 2, right) was fitted with three equidistant tactors and was worn at the front and back of the forearm. The straight layout applies to the more general case of encoding real-valued scalars within a bounded range.

\section{Procedure}

Each participant tested both display layouts (counterbalanced start with either circular or straight layout) in both positions (counterbalanced start in either position) on their nondominant (left) arm, with four trial conditions in total. Note that the distance between tactors varies with wrist circumference, upper arm circumference, and forearm length of each user.

\section{Introduction Phase}

Each experiment (i.e. each pair of display layout and position) was started with an introduction phase (3-4 min) involving a verbal explanation and a trial of the vibrotactile display. These varied depending on the display layout.

Straight Layout: It was explained that stimulus locations represented percentage values as follows: the tactor position at the wrist corresponded to $0 \%$, the position of the tactor closest to the elbow corresponding to $100 \%$, and values in between increased linearly along the arm. Then, the graphical user interface (GUI) for testing was shown on screen, consisting of a single vertical progress bar (see Fig. 1, right). It was explained that the empty bar corresponded to $0 \%$ the full bar corresponded to $100 \%$. In preparation for the trial run, 80 
values were sampled uniformly. Every value was then stimulated with the corresponding GUI representation shown on the screen. This was to familiarise participants with the vibrotactile display and to test the phantom effect: as a condition to proceed to the test phase, each participant was asked whether they felt one or two stimuli at a time, whether stimuli were also perceived in between tactors, and whether they found a correspondence between the location of the stimuli and the values shown on the screen.

Circular Layout: It was explained that the stimulus location represented a direction as on a compass, where the top tactor corresponded to North, the right tactor corresponded to East, and so forth. Then, the GUI for testing was shown on screen, consisting of a circle with four red dots (see Figure 1, left). It was explained that the top dot corresponded to North, the right dot corresponded to East, and so forth. In preparation for the trial run, 72 values were sampled uniformly. Every value was then stimulated with the corresponding GUI representation shown on the screen, where a marker in the shape of a blue dot appeared at the location corresponding to the stimulus. Participants proceeded to the test phase under the same conditions as explained for the straight layout.

\section{Test Phase}

In preparation for the test phase, the sequence of values was shuffled such that stimuli were applied in a new random order. The test phase uses the exact samples of stimuli as the introduction phase. One stimulus was applied, and participants were asked to mark the value corresponding to the perceived stimulus on the GUI. For straight layouts, users were asked to use the mouse to click on the empty progress bar at the location that corresponded to the stimulus. For circular layouts, users were asked to adjust a blue visual marker initially set at North by moving the mouse and to confirm the location with a click. Participants received the stimulus until their response was confirmed and the next stimulus was applied. For each stimulus, we logged the participant's response and the set of tactor intensities. Upon completion of the test phase, participants continued with the introduction phase for the next combination of layout and position.

\section{Data Preprocessing}

During the experiment, we noticed that the circular display was shifted when participants' hands were supinated or pronated relative to the rest position (see Figure 2, left), introducing a systematic bias in user responses. In order to avoid systematic over-estimation of errors due to this misalignment, we shifted all user inputs by the negative mean error, estimated independently for each user and body position.

\section{Results}

For statistical analysis, we considered two body positions for each layout, circular (C): 1) wrist and 2) upper arm, and straight (S): 1) back of the forearm and 2) front of the forearm, and six perceptual models: power (P), linear (L), log (G), sensitivity-adjusted linear (LS), sensitivity-adjusted power (PS) and sensitivity-adjusted $\log$ (GS). The independent variables were layout $\in\{\mathrm{C}, \mathrm{S}\}$, body position $\in\{1,2\}$ and model $\in\{\mathrm{P}, \mathrm{L}, \mathrm{G}, \mathrm{PS}, \mathrm{LS}$ and GS $\}$.

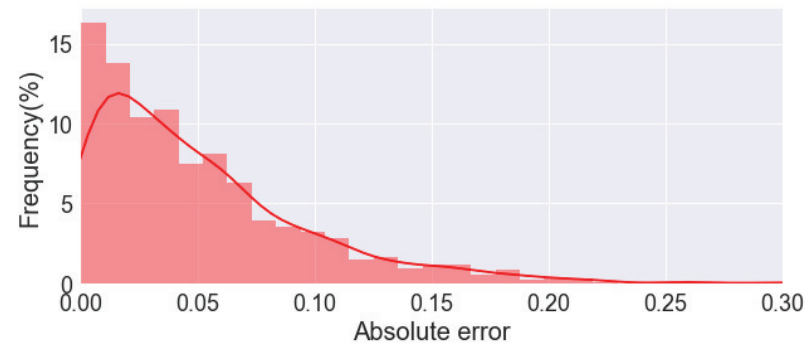

Figure 3: Histogram of absolute error for the wrist position

From each participant's data for a given layout and position, we generated six sets of encoded values $v$, one for each model, by transforming logged tactor intensities to stimulus locations (see Eqns. (2)- (9)). This avoids repeating measurements with participants and justifies paired t-test comparisons of random samples. The data within each combination of user, layout and position was randomly split into equally large training and test sets. The training set was used to infer user and locationspecific sensitivities for sensitivity-adjusted models. The test set was used to evaluate all models. Thus, all the error rates reported below are based on the test set only.

The absolute decoding error was estimated as $\varepsilon=|v-y| \epsilon$ $[0,1]$, where $y \in[0,1]$ is the user response and $v \in[0,1]$ is the encoded value. Within each combination of layout, position, and model, we estimated each participant's central tendency of absolute error. As absolute errors were not normally distributed (see the histogram in Fig. 3), we used the median as the central tendency. This aggregate error was the dependent variable in our analysis. In total, statistical analysis was based on 16 (users) $\times 2$ (layouts) $\times 2$ (positions $) \times 6$ (models $)=$ 384 measurements.

The decoding errors under all tested conditions are given in Table 2 and depicted in Fig. 6. For circular layouts, the power model $(\mathrm{P})$ performed best on average among all generic models, and the sensitivity-adjusted power model (PS) showed lowest mean error overall. For straight layouts, the log model (G) outperformed other generic models on average, and the sensitivity-adjusted log model (GS) best-explained user responses overall. Under every single condition, the sensitivity adjusted model produced a lower mean decoding error compared to the corresponding generic model.

The effects of independent variables on decoding error were analysed using factorial ANOVA. Significant effects were found for layout; $F(1,360)=175.56, p=0.0$, and model; $F(1,360)=4.64, p=0.0$. A significant interaction effect was found between layout and model; $F(1,360)=2.7, p=0.02$. There was no significant interaction effect between layout and gender; $F(1,360)=0.017, p=0.98$, or model and gender; $F(1,360)=1.7, p=0.13$.

We also performed separate factorial ANOVA tests for each layout. With the circular layout, we found a significant effect of body position; $F(1,180)=14.15, p=0.0$, but none of the model; $F(1,180)=1.76, p=0.12$. For the straight layout, 


\begin{tabular}{|c|c|c|c|c|c|c|c|}
\hline \multirow[b]{2}{*}{ Layout } & \multirow[b]{2}{*}{ Position } & \multicolumn{3}{|c|}{ Generic Models } & \multicolumn{3}{|c|}{ Sensitivity-adjusted Models } \\
\hline & & $\varepsilon_{l}$ & $\varepsilon_{p}$ & $\varepsilon_{g}$ & $\varepsilon_{l s}$ & $\varepsilon_{p s}$ & $\varepsilon_{g s}$ \\
\hline \multirow{3}{*}{ Circular } & Upper Arm & $.059(.014)$ & $.054(.014)$ & $.056(.014)$ & $.053(.016)$ & $.051(.014)$ & $.054(.013)$ \\
\hline & Wrist & $.052(.009)$ & $.046(.006)$ & $.049(.008)$ & $.049(.010)$ & $.044(.008)$ & $.048(.010)$ \\
\hline & Both & $.056(.012)$ & $.050(.012)$ & $.053(.011)$ & $.051(.013)$ & $.048(.012)$ & $.051(.012)$ \\
\hline \multirow{3}{*}{ Straight } & Front & $.082(.031)$ & $.082(.026)$ & $.080(.029)$ & $.070(.018)$ & $.074(.022)$ & $.068(.020)$ \\
\hline & Back & $.090(.022)$ & $.085(.027)$ & $.086(.022)$ & $.067(.018)$ & $.073(.027)$ & $.064(.020)$ \\
\hline & Both & $.086(.027)$ & $.084(.026)$ & $.083(.026)$ & $.069(.018)$ & $.074(.024)$ & $.066(.020)$ \\
\hline
\end{tabular}

Table 2: Decoding error (M and SD) for each layout, position and model. Notation: $\varepsilon_{l}$ - linear model, $\varepsilon_{p}$ - power model, $\varepsilon_{g}$ - log model, $\varepsilon_{l s}$ - sensitivity-adjusted linear model, $\varepsilon_{p s}$ - sensitivity-adjusted power model and $\varepsilon_{g s}$ - sensitivity-adjusted log model.

\begin{tabular}{c|c|c|c|c|c|c|c|c|c} 
Layout & $\varepsilon_{l}$ vs $\varepsilon_{p}$ & $\varepsilon_{l}$ vs $\varepsilon_{g}$ & $\varepsilon_{p}$ vs $\varepsilon_{g}$ & $\varepsilon_{l}$ vs $\varepsilon_{l s}$ & $\varepsilon_{p}$ vs $\varepsilon_{p s}$ & $\varepsilon_{g}$ vs $\varepsilon_{g s}$ & $\varepsilon_{l s}$ vs $\varepsilon_{p s}$ & $\varepsilon_{l s}$ vs $\varepsilon_{g s}$ & $\varepsilon_{p s}$ vs $\varepsilon_{g s}$ \\
\hline Circular & $\mathbf{0 . 0 0 1}$ & $\mathbf{0}$ & $\mathbf{0 . 0 0 8}$ & $\mathbf{0 . 0 0 6}$ & 0.205 & 0.175 & 0.069 & 0.925 & $\mathbf{0 . 0 3 2}$ \\
\hline Straight & 0.603 & $\mathbf{0 . 0 0 8}$ & 0.697 & $\mathbf{0 . 0 0 1}$ & $\mathbf{0 . 0 1}$ & $\mathbf{0 . 0 0 1}$ & 0.116 & 0.116 & $\mathbf{0 . 0 0 6}$ \\
\hline
\end{tabular}

Table 3: Statistical significance in p-values of paired t-tests.

body position had no significant effect; $F(1,180)=0.20, p=$ 0.65 , but the model did; $F(1,180)=4.12, p=0.001$.

The results of posthoc paired t-tests are presented in Table 3. Comparing generic models on the circular layout, all models differed significantly from each other, whereas on the straight layout only a significant difference between the linear model and the log model was found. Comparing generic and sensitivity-adjusted models on the circular layout, only the linear model showed a significant difference. On the straight layout, all sensitivity-adjusted models showed significantly lower error than corresponding generic models. Among sensitivityadjusted models, the power model and the log model differed significantly with both layouts.

Inspecting the decoding error observed with circular layouts and the sensitivity-adjusted power model, there was no significant difference between body positions; $t(32)=2.04, p=$ 0.058. Equally, there was no significant difference between body positions; $t(32)=0.65, p=0.52$ among straight layouts with sensitivity-adjusted log model.

Pearson correlation analysis confirmed that there was no significant correlation between the error on the straight layout (using GS) and the forearm size; $r=0.21, p=0.22$. Similarly, there was neither a significant correlation between the error on the upper arm display (with PS) and the circumference of the upper arm $r=-0.07, p=0.77$ nor the wrist display the circumference of the wrist $r=-0.35, p=0.17$.

\section{DISCUSSION}

Our study demonstrated that paricipants can decode a realvalued values with a mean error of only $4.4 \%$ for circular displays (on the wrist) and $6.4 \%$ for straight displays (on the back of the forearm). This low error was achieved with the proposed method for personalisation of perceptual models for tactile displays using sensitivity adjustment. The proposed method consistently outperformed the corresponding generic model with regards to decoding error of real-valued data. This improvement was significant for the best model (the $\log$ model) for straight layouts, validating our approach. We have shown that error-reducing local perceptual sensitivities can be inferred from user data. An example of how a linear

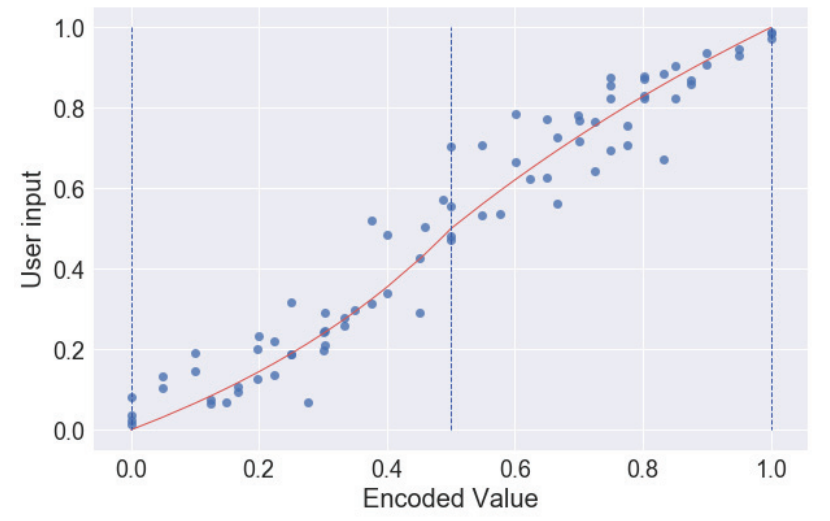

Figure 4: The sensitivity-adjust linear model (continuous red line) approximates the user's perception of the encoded value on a straight display. The $x$-axis represents the encoded value using a linear model and the dotted lines represent positions of tactors.

sensitivity-adjusted model approximates user's perception is presented in Figure 4.

Wrist-based circular displays showed lower decoding error than displays worn around the upper arm. However, there are practical aspects that differentiate those displays further. Wrist-based displays may be easier to offer as mainstream products (e.g. alongside smartwatches). The potential for movement around the wrist can, however, pose problems. The user might interpret values differently when decoding values from a rotated display, perhaps relative to the body position. This could be avoided by using motion sensors on the wrist to detect and compensate for the orientation of the wrist when encoding a value. Such sensors are typically available on wristbands, but determining the orientation is prone to error. This problem does not occur on the upper arm as pronation and supination are not physically possible.

Similarly, we observed higher accuracy on the back of the forearm than at the front, but it is often in contact and quite often attached to the body. This could affect the perception of vibration if the motors are pushed towards the body. 


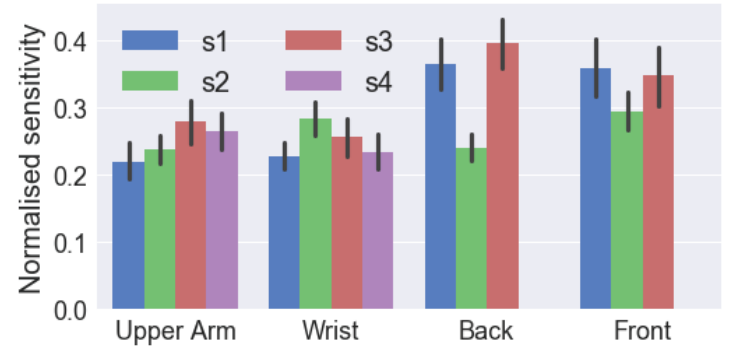

Figure 5: Normalised optimal user sensitivities

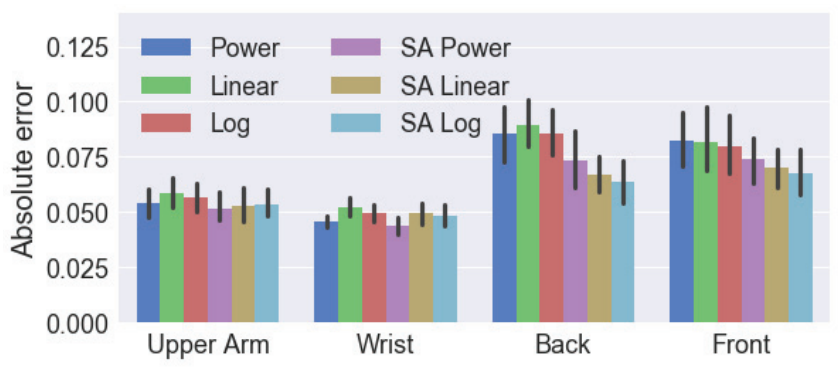

Figure 6: Absolute errors for each layout, position and model

The circular layout performed significantly better than the straight layout. To some degree that is not a fair comparison, as the former had four and the later had three tactors. Depending on the application and the nature of encoded values, one layout may be better suited than the other (and vice-versa). For the applications representing a state of a circular or repetitive processes (e.g. direction guide, representing an angle, the progress of a lap in a racing game) the circular display would be a great choice. For other applications, the proximity of two extreme values in a circular layout and the risk of them being mistaken for each other would make it an impractical choice.

Figure 5 presents the sensitivities of locations for each display averaged among all users. Note that as the ratio between $s_{i}$ values within $S$ is important, the sensitivities are normalised within $S\left(\sum_{i} s_{i}=1\right)$. Focusing on the front and back displays, we see that areas near the wrist and the upper part of the forearm are more sensitive than the middle part. This is in line with findings on vibrotactile localisation on the forearm by Cholewiak [9]. There is considerable variation across participants (visualised by black lines) at each location. This could be attributed to idiosyncratic variations of sensitivity levels. In addition to skin sensitivity, factors influencing how strong a vibration is perceived include how tight a tactor is attached to the skin, tactor orientation and manufacturing inaccuracies, especially for cheaper devices. Thus, the test equipment can directly affect the model.

As presented in the Tables 2 and 3 the sensitivity adjusted models outperform generic models in predicting the user perceived value by capturing different levels of sensitivity of the locations, personal sensitivity variations and the device (tactor) characteristics within a small set of parameters $\left(s_{i}\right)$. It is interesting that, on circular layouts, the sensitivity-adjusted power model is most accurate, whereas, on straight layouts, the sensitivity-adjusted log model performed best. As there is no closed form solution to predicting locations with the log model, the process of computing optimal sensitivities is computationally expensive. However, once the sensitivity parameters $S_{o}$ are estimated, encoding values can done in closed form for all models (Eqns. 6, 9 and 7). Thus, we do not expect this to be an important issue in most of the cases.

There is room for further improvement on the decoding error rate. In our study participants used such a display for the first time. Long term use and feedback on user performance is expected to result in better perception and recognition of encoded values. Other haptic related studies provide evidence of learning effects when using vibrotactile devices for longer duration [22, 27].

Another important direction for future work is quantifying the ease of use, setup time, and decoding accuracy with different manual and automatic calibration methods.

\section{CONCLUSION}

In this paper, we investigated user decoding precision of spatially modulated real-valued data, which was decoded by localising spatially continuous, temporally stationary vibrotactile stimuli. In a user study, we quantified decoding precision using six perceptual models across two vibrotactile display layouts.

We developed personalised sensitivity-adjusted perceptual models for tactile displays based on the linear, log and power models. We have shown that the proposed models consistently outperform corresponding generic models, increasing the accuracy of decoding information by participants. The best sensitivity-adjusted models approximated users' perception with an average error of only $6.4 \%$ for straight display layouts and only $4.4 \%$ for circular display layouts.

Increasing decoding accuracy has the potential to improve the sense of realism and acceptance by users, and facilitate new applications of tactile displays in a wide range of real-world application areas.

\section{ACKNOWLEDGMENTS}

This work was funded by MoreGrasp (H2020-ICT-2014-1 643955). The Know-Center is funded within the Austrian COMET Program - Competence Centers for Excellent Technologies - under the auspices of the Austrian Federal Ministry of Transport, Innovation and Technology, the Austrian Federal Ministry of Economy, Family and Youth and by the State of Styria. COMET is managed by the Austrian Research Promotion Agency FFG.

\section{REFERENCES}

1. Alles, D. S. Information transmission by phantom sensations. IEEE Transactions on Man-Machine Systems 11, 1 (March 1970), 85-91.

2. Badke, M. B., Sherman, J., Boyne, P., Page, S., and Dunning, K. Tongue-based biofeedback for balance in stroke: results of an 8-week pilot study. Archives of physical medicine and rehabilitation.

3. Barghout, A., Cha, J., Saddik, A. E., Kammerl, J., and Steinbach, E. Spatial resolution of vibrotactile perception on the human forearm when exploiting funneling illusion. In 2009 IEEE International Workshop on Haptic Audio visual Environments and Games (Nov 2009), 19-23.

4. Bliss, J. C., Katcher, M. H., Rogers, C. H., and Shepard, R. P. Optical-to-tactile image conversion for the blind. IEEE Transactions on Man-Machine Systems 11, 1 (March 1970), 58-65. 
5. Brown, L. M., and Kaaresoja, T. Feel who's talking: Using tactons for mobile phone alerts. In CHI'O6 Extended Abstracts on Human Factors in Computing Systems, CHI EA '06, ACM (2006), 604-609.

6. Byrd, R. H., Lu, P., Nocedal, J., and Zhu, C. A Limited Memory Algorithm for Bound Constrained Optimization. SIAM J. Sci. Comput. 16, 5 (Sept. 1995), 1190-1208.

7. Cha, J., Rahal, L., and Saddik, A. E. A pilot study on simulating continuous sensation with two vibrating motors. In 2008 IEEE International Workshop on Haptic Audio visual Environments and Games (Oct 2008), 143-147.

8. Chatterjee, A., Chaubey, P., Martin, J., and Thakor, N. V. Quantifying prosthesis control improvements using a vibrotactile representation of grip force. In 2008 IEEE Region 5 Conference (April 2008), 1-5.

9. Cholewiak, R. W., and Collins, A. A. Vibrotactile localization on the arm: Effects of place, space, and age. Perception \& Psychophysics (2003).

10. Crossan, A., and Brewster, S. Two-handed navigation in a haptic virtual environment. In CHI '06 Extended Abstracts on Human Factors in Computing Systems, CHI EA '06, ACM (New York, NY, USA, 2006).

11. de Jesus Oliveira, and Maciel, A. Assessment of tactile languages as navigation aid in 3d environments. In EUROHAPTICS, 2014 (2014).

12. de Jesus Oliveira, and Maciel, A. Introducing the modifier tactile pattern for vibrotactile communication. In EUROHAPTICS, 2014 (2014).

13. Ding, Z. Q., Luo, Z. Q., Causo, A., Chen, I. M., Yue, K. X., and Yeo, S. H. Inertia sensor-based guidance system for upperlimb posture correction. Med Eng Phys 35 (2013).

14. Eid, M., Korres, G., and Jensen, C. B. F. Soa thresholds for the perception of discrete/continuous tactile stimulation. In 2015 Seventh International Workshop on Quality of Multimedia Experience (QoMEX) (May 2015), 1-6.

15. Gault, R. H. Progress in experiments on tactual interpretation of oral speech. The Journal of Abnormal Psychology and Social Psychology 19, 2 (1924), 155

16. Geldard, F. A. Adventures in tactile literacy. American Psychologist 12, 3 (1957), 115-124.

17. Gopalai, A. A., and Senanayake, S. M. N. A. A wearable real-time intelligent posture corrective system using vibrotactile feedback. IEEE Trans Mechatronics 16 (2011).

18. Gunther, E. Skinscape: A tool for composition in the tactile modality. $\mathrm{PhD}$ thesis, Massachusetts Institute of Technology, 2001.

19. Israr, A., Kim, S.-C., Stec, J., and Poupyrev, I. Surround haptics: Tactile feedback for immersive gaming experiences. In CHI'12 Extended Abstracts on Human Factors in Computing Systems, CHI EA '12 (2012).

20. Israr, A., and Poupyrev, I. Control space of apparent haptic motion. In 2011 IEEE World Haptics Conference (June 2011), 457-462.

21. Israr, A., and Poupyrev, I. Tactile brush: Drawing on skin with a tactile grid display. In Proceedings of the SIGCHI Conference on Human Factors in Computing Systems, CHI '11 (2011).

22. Kaul, O. B., and Rohs, M. Haptichead: A spherical vibrotactile grid around the head for $3 \mathrm{~d}$ guidance in virtual and augmented reality. In Proceedings of the 2017 CHI Conference on Human Factors in Computing Systems, CHI' 17 (2017).

23. Kentala, E., Vivas, J., and Conrad Wall, I. Reduction of postural sway by use of a vibrotactile balance prosthesis prototype in subjects with vestibular deficits. Annals of Otology, Rhinology \& Laryngology 112.

24. Kirman, J. H. Tactile perception of computer-derived formant patterns from voiced speech. The Journal of the Acoustical Society of America (1974).

25. Lee, J., Kim, Y., and Kim, G. J. Effects of visual feedback on out-of-body illusory tactile sensation when interacting with augmented virtual objects. IEEE Transactions on Human-Machine Systems 47, 1 (Feb 2017), 101-112

26. Liao, Y.-C., Chen, Y.-L., Lo, J.-Y., Liang, R.-H., Chan, L., and Chen, B.-Y. Edgevib: Effective alphanumeric character output using a wrist-worn tactile display. In Proceedings of the 29th Annual Symposium on User Interface Software and Technology, UIST '16 (2016).

27. Luzhnica, G., Veas, E., and Pammer, V. Skin reading: Encoding text in a 6-channel haptic display. In Proceedings of the 2016 ACM International 73 Symposium on Wearable Computers, ISWC'16 (2016).
28. Mackinlay, J. Automating the design of graphical presentations of relational information. ACM Transactions on Graphics 5, 2 (Apr. 1986).

29. Makous, J. C., Friedman, R. M., and Vierck, C. J. A critical band filter in touch. The Journal of neuroscience : the official journal of the Society for Neuroscience 154 (1995), 2808-18.

30. Nicolau, H., Guerreiro, J. a., Guerreiro, T., and Carriço, L. Ubibraille Designing and evaluating a vibrotactile braille-reading device. In ACM SIGACCESS, ASSETS '13 (2013).

31. Novich, S. D., and Eagleman, D. M. Using space and time to encode vibrotactile information: toward an estimate of the skin's achievable throughput. Experimental Brain Research, 10 (2015).

32. Ooka, T., and Fujita, K. Virtual object manipulation system with substitutive display of tangential force and slip by control of vibrotactile phantom sensation. In 2010 IEEE Haptics Symposium (2010).

33. Park, J., Kim, J., Oh, Y., and Tan, H. Z. Rendering moving tactile stroke on the palm using a sparse 2d array. In Haptics: Perception, Devices, Control, and Applications:International Conference, EuroHaptics 2016, Springer International Publishing (Cham, 2016).

34. Pylatiuk, C., Kargov, A., and Schulz, S. Design and evaluation of a low-cost force feedback system for myoelectric prosthetic hands. JPO: Journal of Prosthetics and Orthotics 18, 2 (2006), 57-61.

35. Rahal, L., Cha, J., and Saddik, A. E. Continuous tactile perception for vibrotactile displays. In 2009 IEEE International Workshop on Robotic and Sensors Environments (Nov 2009), 86-91.

36. Rosenthal, J., Edwards, N., Villanueva, D., Krishna, S., McDaniel, T., and Panchanathan, S. Design, implementation, and case study of a pragmatic vibrotactile belt. IEEE Transactions on Instrumentation and Measurement 60, 1 (Jan 2011), 114-125.

37. Saunders, I., and Vijayakumar, S. The role of feed-forward and feedback processes for closed-loop prosthesis control. Journal of NeuroEngineering and Rehabilitation 8, 1 (2011), 60.

38. Schneider, O. S., Israr, A., and MacLean, K. E. Tactile animation by direct manipulation of grid displays. In Proceedings of the Annual ACM Symposium on User Interface Software \& Technology, UIST' 15 (2015).

39. Seo, J., and Choi, S. Initial study for creating linearly moving vibrotactile sensation on mobile device. In 2010 IEEE Haptics Symposium (March 2010), 67-70.

40. Shull, P. B., and Damian, D. D. Haptic wearables as sensory replacement, sensory augmentation and trainer - a review. Journal of NeuroEngineering and Rehabilitation 12, 1 (2015), 59.

41. Spence, C., and Ho, C. Tactile and multisensory spatial warning signals for drivers. Haptics, IEEE Transactions on 1, 2 (July 2008), 121-129.

42. Summers, I. R., Whybrow, J. J., Gratton, D. A., Milnes, P., Brown, B. H., and Stevens, J. C. Tactile information transfer: A comparison of two stimulation sites. The Journal of the Acoustical Society of America 118.

43. Tan, H. Z., Gray, R., Young, J., and Traylor, R. A haptic back display for attentional and directional cueing. Journal of Haptics Research 3 (2003).

44. Ternes, D., and MacLean, K. E. Designing large sets of haptic icons with rhythm. In EuroHaptics, M. Ferre, Ed. (2008).

45. Tsukada, K., and Yasumura, M. ActiveBelt: Belt-Type Wearable Tactile Display for Directional Navigation. Springer, Berlin, Heidelberg, 2004.

46. Ueda, S., Uchida, M., Nozawa, A., and Ide, H. A tactile display used phantom sensation with apparent movement together. Electronics and Communications in Japan, Part II: Electronics (2008).

47. Wall, C., and Weinberg, M. S. Balance prostheses for postural control. IEEE Engineering in Medicine and Biology Magazine 22, 2 (2003).

48. White, B. W., Saunders, F. A., Scadden, L., Bach-Y-Rita, P., and Collins, C. C. Seeing with the skin. Perception \& Psychophysics 7, 1, 23-27.

49. Xu, C., Israr, A., Poupyrev, I., Bau, O., and Harrison, C. Tactile display for the visually impaired using teslatouch. In $\mathrm{CHI}$ ' 11 Extended Abstracts on Human Factors in Computing Systems, CHI EA '11 (2011).

50. Yang, G. H., Ryu, D., Park, S., and Kang, S. Sensory saltation and phantom sensation for vibrotactile display of spatial and directional information. Presence 21, 2 (Feb 2012), 192-202.

51. Yannier, N., Israr, A., Lehman, J. F., and Klatzky, R. L. FeelSleeve: Haptic feedback to enhance early reading. In Proceedings of Annual ACM Conference on Human Factors in Computing Systems, CHI (2015). 